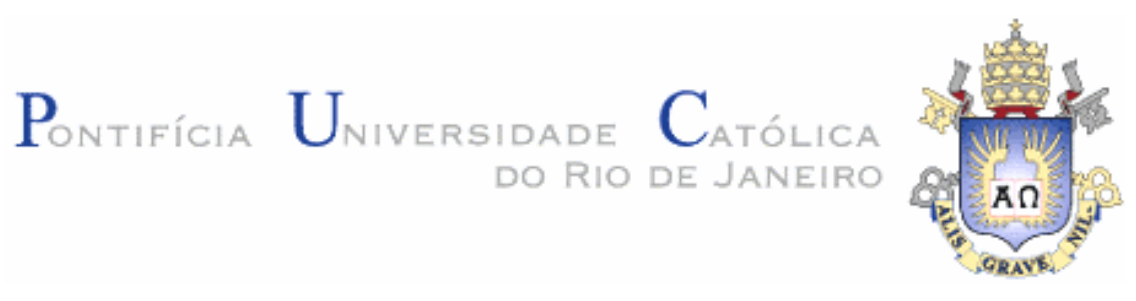

Leonardo Rodin Salas Cachay

Fluxo de Partículas de Sustentação em Poços de Petróleo Estimulados por Fraturamento Hidráulico

Dissertação de Mestrado

Dissertação apresentada como requisito parcial para obtenção do título de Mestre pelo Programa de PósGraduação em Engenharia Civil da PUC-Rio. Área de Concentração: Geotécnia

Orientador: Celso Romanel.

Co-orientador: Araken Dumont Ramos Lima

Rio de Janeiro, Dezembro de 2004 


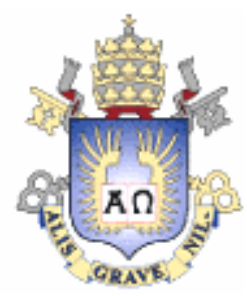

Leonardo Rodin Salas Cachay

\title{
Fluxo de Partículas de Sustentação em Poços de Petróleo Estimulados por Fraturamento Hidráulico
}

Dissertação apresentada como requisito parcial para obtenção do título de Mestre pelo Programa de PósGraduação em Engenharia Civil da PUC-Rio. Aprovada pela Comissão Examinadora abaixo assinada.

\author{
Celso Romanel \\ Orientador \\ Departamento de Engenharia Civil - PUC-Rio
}

Araken Dumont Ramos Lima Co-orientador

Departamento de Engenharia Civil - PUC-Rio

Paulo Dore Fernandes CENPES/PETROBRAS

Deane Mesquita Roehl Departamento de Engenharia Civil - PUC-Rio

José Eugenio Leal

Coordenador Setorial do Centro

Técnico Científico - PUC-Rio

Rio de Janeiro, Dezembro de 2004 
Todos os direitos reservados. É proibida a reprodução total ou parcial do trabalho sem autorização da universidade, do autor e do orientador.

\section{Leonardo Rodin Salas Cachay}

Graduou-se em Engenharia Civil na UNI (Universidad Nacional de Ingeniería - Peru) em 2001. Ingressou no curso de Mestrado em Engenharia Civil da PUC-Rio em 2002.2, desenvolvendo estudos na linha de pesquisa Geomecânica Computacional.

Ficha Catalográfica

\section{Salas Cachay, Leonardo Rodin}

Fluxo de partículas de sustentação em poços de petróleo estimulados por fraturamento hidráulico / Leonardo Rodin Salas Cachay; orientador: Celso Romanel; co-orientador: Araken Dumont Ramos Lima. - Rio de Janeiro: PUC, Departamento de Engenharia Civil, 2004.

v., 132 f.: il. ; $29.7 \mathrm{~cm}$

Dissertação (mestrado) - Pontifícia Universidade Católica do Rio de Janeiro, Departamento de Engenharia Civil.

Inclui referências bibliográficas.

1. Engenharia Civil - Teses. 2. Refluxo de propante. 3. Propriedades dos propantes. 4. Poços de petróleo. 5. Fraturamento hidráulico. 6. Modelos experimentais. I. Romanel, Celso. II. Ramos Lima, Araken Dumont. III. Pontifícia Universidade Católica do Rio de Janeiro. Departamento de Engenharia Civil. IV. Título. 


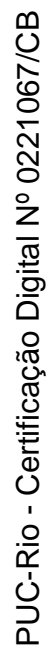

A meus pais e irmãos, à Sonia, minha esposa, por terem confiado em mim. 


\section{Agradecimentos}

A meus pais e irmãos, pela atenção, carinho e ensinamentos, sem os quais eu não teria chegado até aqui.

Ao professor Celso Romanel, pela paciência, confiança depositada, orientação, estímulo e conhecimentos transmitidos.

Ao Araken Dumont Ramos Lima pela participação em momentos decisivos desta dissertação.

Aos professores do Departamento de Engenharia Civil da PUC - Rio.

A todos os amigos, especialmente os da sala 317-L e 607-C, pelo apoio nas horas difíceis.

Aos funcionários do Departamento de Engenharia Civil da PUC-Rio..

À PUC e à Capes pelos recursos financeiros que possibilitaram o desenvolvimento da pesquisa.

Ao Cenpes/Petrobrás, em especial ao engenheiro Paulo Dore Fernandes, pelo fornecimento dos dados relativos aos poços de petróleo de Sergipe.

A Javier Cânon e Max Salas pela colaboração que, de alguma forma, também contribuíram para o sucesso desta pesquisa.

À minha família no Peru e aos amigos que se preocuparam em manter os laços de amizade e de carinho apesar da longa distância

Aos meus primos Hada e Martin pelo incentivo e suporte que sempre me deram. 


\section{Resumo}

Salas Cachay, Leonardo Rodin. Romanel, Celso. Ramos Lima, Araken Dumont. Fluxo de partículas de sustentação em poços de petróleo estimulados por fraturamento hidráulico. Rio de Janeiro, 2004. 132p. Dissertação de Mestrado - Departamento de Engenharia Civil, Pontifícia Universidade Católica do Rio de Janeiro.

Um dos principais objetivos da engenharia de petróleo é desenvolver e aplicar técnicas capazes de aumentar a produtividade de poços de petróleo, incluindo a estimulação da formação através de operações que aumentem a permeabilidade da rocha-reservatório e facilitem o escoamento do fluido. Dentre as técnicas de estimulação, a mais utilizada é o fraturamento hidráulico, a qual tem viabilizado a exploração em cerca de $40 \%$ dos poços produtores de petróleo em todo o mundo. Durante o fraturamento hidráulico, um material granular conhecido como material de sustentação ou propante, é injetado nas fraturas recém-criadas com o objetivo de mantê-las abertas e garantir-lhes condições de alta permeabilidade. A produção de material de sustentação (proppant flowback) é termo usado para descrever o refluxo do propante para o interior do poço, juntamente com o hidrocarboneto produzido. O controle do fluxo de propante representa um grave desafio para a indústria de petróleo, pois pode causar graves problemas operacionais e de segurança, relacionados com o desgaste dos equipamentos de produção, problemas econômicos associados ao custo de limpeza, paralisação das operações e intervenção no tratamento da fratura, problemas ambientais relacionados com a disposição de sólidos impregnados por hidrocarbonetos, etc. Neste contexto, a presente dissertação apresenta um estudo detalhado dos principais tipos de propante e de suas propriedades, bem como descreve os mecanismos que influem no refluxo do material de sustentação da fratura e analisa os principais modelos apresentados na literatura para previsão deste fenômeno. Uma retroanálise considerando os dados de campo em 22 poços

da Petrobrás, localizados em Sergipe, permite uma comparação da eficiência entre quatro dos modelos, desenvolvidos com base em resultados de ensaios de laboratório, indicando suas vantagens e desvantagens para aplicação na prática da engenharia.

\section{Palavras-chave}

Refluxo de propante; propriedades dos propantes; poços de petróleo; fraturamento hidráulico; modelos experimentais. 


\section{Abstract}

Salas Cachay, Leonardo Rodin. Romanel, Celso \& Ramos Lima, Araken Dumont (Advisors). Proppant flowback in oil wells stimulated by hydraulic fracturing. Rio de Janeiro, 2004. 132p. MSc. Thesis Department of Civil Engineering, Pontifícia Universidade Católica do Rio de Janeiro.

One of the main objectives of petroleum engineering is to develop and to apply techniques aiming the productivity increase of oil fields, including the stimulation of the rock through operations that increase the permeability of the oil reservoir and makes the flow of the fluid toward the well more efficient. Among the stimulation techniques, the most used is the hydraulic fracturing, carried out in about $40 \%$ of the producing oil wells around the world. During hydraulic fracturing, a granular material known as proppant, is injected into the just created fractures with the objective to keep them open and in order to guarantee the designed conditions of high permeability. Proppant flowback is the technical terminology employed to describe the flow of proppant from the fracture to the interior of the oil well, together with the produced hydrocarbon. An efficient and reliable control of this problem is still a major challenge to the oil industry, given the serious operational and security problems that it may cause, including costs of interrupted operations, cleaning measures, environmental contamination risks associated with the disposal of residues impregnated by oil, etc. In this context, the present thesis presents a detailed description on the main proppant materials and their engineering properties and available treatments, as well as on the physical mechanisms that control the flowback phenomenon. Several simple models published in the literature, that permit the forecast of proppant flowback in a fast and easy way, are also presented and discussed. Finally, a back-analysis considering the actual conditions in 22 Petrobras oil wells located in Sergipe was also carried out, what permitted a comparison among the results calculated according 4 prediction models, putting in evidence their advantages, shortcomings and adequacy as a design or control tool in the design and exploration of oil fields stimulated by hydraulic fracturing.

\section{Keywords}

Proppant flowback; proppant properties; oil wells; hydraulic fracturing; experimental models. 


\section{Sumário}

1 INTRODUÇÃO

2 MATERIAIS DE SUSTENTAÇÃO DE FRATURAS 26

2.1. PRINCIPAIS TIPOS DE PROPANTES 26

2.1.1. Propriedades físicas dos propantes 27

2.1.1.1. Resistência ao esmagamento 28

2.1.1.2. Tamanho e distribuição dos grãos 28

2.1.1.3. Arredondamento e esfericidade das partículas 30

2.1.1.4. Densidade do propante 31

2.2. TRATAMENTOS DE PROPANTES 32

2.2.1. Tratamento com resina 32

2.2.2. Lavagem com resina 34

2.2.3. Agentes modificadores de superfície 34

2.2.4. Aditivos sólidos: fibras $\quad 35$

2.2.5. Aditivos sólidos: filme termoplástico 36

2.2.6. Aditivos sólidos: DIP 37

3 REFLUXO DO MATERIAL DE SUSTENTAÇÃO 41

3.1. INTRODUÇÃO 41

3.2. LARGURA DA FRATURA 44

3.3. TENSÃO DE FECHAMENTO DA FRATURA 46

3.4. FORÇAS DE ARRASTE 50

3.4.1. Efeitos do fluxo não-Darciano 53

3.4.2. Efeitos do fluxo multifásico

3.5 CARACTERÍSTICAS DO PROPANTE 56

3.6 EFEITOS DA GRAVIDADE E DA VISCOSIDADE 59

3.7 FECHAMENTO FORÇADO DA FRATURA 60

3.8. EFEITO DA DEPLEÇÃO DO RESERVATÓRIO 60 
4 MODELOS PARA PREVISÃO DE REFLUXO DO MATERIAL DE SUSTENTAÇÃO DA FRATURA

4.1. MODELOS EMPÍRICOS 61

4.1.1. Modelo do consórcio Stimlab 61

4.1.2. Modelo da cunha livre 66

4.1.3. Modelo de Potência 69

4.1.4. Modelo da Correlação de Bi-Potência 73

4.2. MODELOS TEÓRICOS

4.2.1. Modelo da velocidade mínima de fluidificação 75

4.2.2. Modelo Semi-Mecânico

4.2.3. Método dos Elementos Discretos 81

4.3. CASO HISTÓRICO - ESTUDO COMPARATIVO ENTRE OS MODELOS EMPÍRICOS

5 RETROANÁLISE DE CASO HISTÓRICO 89

5.1. CARACTERÍSTICAS DOS POÇOS DA PETROBRÁS 89

5.2. PROPRIEDADES DO FLUIDO 90

5.2.1 Densidade 90

5.2.2. Viscosidade 92

5.3. CARACTERÍSTICAS DE PROJETO DO FRATURAMENTO HIDRÀULICO 93

5.4. MODELOS UTILIZADOS NAS RETROANÁLISES 97

5.5. RESULTADOS OBTIDOS 99

5.6. ANÁLISE COMPARATIVA DOS RESULTADOS 109

5.7. PARÃMETROS NECESSÁRIOS PARA ANÁLISE DO REFLUXO 116

5.7.1 Quantidades calculadas neste estudo 118

6 CONCLUSÕES E SUGESTÕES 120

7 REFERÊNCIAS BIBLIOGRÁFICAS 124 


\section{Lista de figuras}

Figura 1.1 - Estatísticas do fraturamento de poços nos Estados Unidos (1945 - 1993) - S. Haidar (2003).

Figura 1.2a - Representação do processo de fraturamento hidráulico (EPA, 2004).

Figura 1.2b - Representação de injeção de fluido contendo propante (EPA, 2004).

Figura 1.2c - Representação o processo de limpeza e produção do poço. (EPA, 2004)

Figura 2.1 - Seleção do tipo de propante de acordo com a tensão de fechamento da fratura (Economides et al., 2000).

Figura 2.2 - Comparação entre os valores de resistência ao esmagamento de vários tipos de propante (Economides, 2000).

Figura 2.3 - Valores de condutividade da fratura em função do tamanho dos grãos de propante e da tensão de fechamento (apud www.carboceramics.com).

Figura 2.4 - Fator de forma de Krumbein para caracterização do arredondamento e da esfericidade de grãos de propantes (RP-60, RP-58, RP-56).

Figura 2.5 - Geometria do arco de compressão em fraturas tratadas com propante e fibras (Romero e Feraud, 1996).

Figura 2.6 - Partícula DIP antes (esquerda) e após (direita) ao fechamento da fratura sob tensão de 7000 psi (48MPa). Os círculos indicam deformação causada pela penetração do propante.

Figura 2.7 - Aumento da resistência ao refluxo do material de sustentação com adição de $15 \%$ de DIP (em peso). Propante cerâmico 20/40 de baixa densidade (Lt. Wt), tensão confinante de 4.000 psi (27 MPa), temperatura de $250^{\circ} \mathrm{F}\left(120^{\circ} \mathrm{C}\right)$ - Rae, P. et al, 2001.

Figura 2.8 - Aumento da permeabilidade do propante pela adição de DIP (Rae, P. et al, 2001).

Figura 3.1 - Fraturamento hidráulico de um poço vertical (Economides, 
2000).

Figura 3.2 - Injeção de propante na fratura (Economides, 2000).

Figura 3.3 - Refluxo do agente de sustentação da fratura hidráulica 1 para o interior do poço de petróleo.

Figura 3.4 - Efeitos da tensão de fechamento na estabilidade de fratura hidráulica. (Canon, 2003)

Figura 3.5 - llustração da penetração do propante na rocha da formação.

(R.D. Barred. Et. Al. 2003).

Figura 3.6 - Esmagamento e penetração do propante na rocha da formação (apud Legarth et al., 2003).

Figura 3.7 - Condutividade da fratura a longo prazo em função da concentração de propante (apud www.carboceramics.com).

Figura 3.8 - Determinação experimental de valores de concentração para assegurar estabilidade do pacote granular, considerando tensão de fechamento da fratura no intervalo $2000 \mathrm{psi}-4500 \mathrm{psi}(13,67 \mathrm{MPa}-$ $30,75 \mathrm{MPa}$ ) na temperatura $120^{\circ} \mathrm{C}-$ (Haidar, 2003).

Figura 3.9 - Fator inercial $\beta$ para propantes de areia considerando vários fluidos: gás, óleo e solução de água e sal (brine) www.carboceramics.com.

Figura 3.10 - Variação do fator $\beta$ para vários tipos de propante (www.carboceramics.com).

Figura 3.11 - Gráfico $\gamma \times x_{t} / x_{t}$ para areia Jordan 20/40 (tensão de fechamento 2000 psi, temperatura $75^{\circ} \mathrm{F}$, pressão interna $100 \mathrm{psi}$ ) www.carboceramics.com

Figura 3.12 -Tensão efetiva no agente de sustentação

Figura 4.1 - Seção longitudinal do pacote granular mostrando as zonas de refluxo (Stimlab, 1996-2002).

Figura 4.2 - Representação gráfica do modelo Stimlab delimitando regiões de estabilidade para diferentes números de camadas de grãos de propante no interior da fratura (Stimlab, 1996-2002).

Figura. 4.3 - Representação gráfica do modelo da cunha livre (Andrews e Kjorholt, 1998). 
Figura 4.4 - Resultados experimentais de ensaios de laboratório (J.Canon, 2003).

Figura 4.5 - Resultados experimentais de laboratório com fluxo monofásico em propantes com abertura de malha 16/30 (Barree e Conway, 2002. 71

Figura 4.6 - Resultados experimentais de laboratório com fluxo monofásico em propantes com abertura de malha 12/20 (Barree e Conway, 2002). 71

Figura 4.7 - Sumário das curvas ajustadas pelo modelo de potência (Barree e Conway, 2002

Fig 4.8 - Sumário das curvas ajustadas pelo modelo de potência com a inclusão de um limite inferior para velocidade de fluxo (Barree e Conway, 2002).

Figura. 4.9 - Envoltórias para areia na malha 20/40.

Figura. 4.10 - Envoltórias para propante cerâmico de alta resistência na malha $20 / 40$.

Figura 4.11 - Retroanálise da estabilidade das fraturas com o modelo Stimlab (Canon et al., 2003)

Figura 4.12 - Retroanálise da estabilidade das fraturas com o modelo semi-mecânico (Canon et al., 2003)

Figura 4.13- Retroanálise da estabilidade das fraturas com o modelo de cunha livre (Canon et al., 2003)

Figura 4.14 - Ilustração de do estrangulamento da fratura junto ao poço ("choked-fracture skin") devido ao refluxo do material de sustentação, (Diego J. Romero, 2003).

Figura 4.15 - Comparações dos índices de produtividade considerando o modelo da cunha livre (Canon et. al, 2003).

Figura 4.16 - Comparações dos índices de produtividade considerando o modelo de Stimlab (Canon et. al, 2003).

Figura 4.17 - Comparações dos índices de produtividade considerando o modelo semi-mecânico (Canon et. al, 2003).

Figura 4.18 - Metodologia para incorporar um critério de estabilidade no projeto do fraturamento hidráulico (Canon et. al, 2003).

Figura 5.1 - Porcentagem do propante produzido em relação ao peso total injetado nos poços da Petrobrás em Sergipe. 
Figura 5.2 - Representações esquemáticas do reservatório e da fratura, (Diego J. Romero,e Peter P. Valkó, 2003)

Figura 5.3 - Índice adimensional de produtividade $J_{D}$ em função do coeficiente adimensional de condutividade da fratura $C_{f D}$ e da razão de penetração $I_{x}$.

Figura 5.4 - Índice adimensional da produtividade em função do coeficiente adimensional de condutividade da fratura e do número de propante para $N_{P} \leq 0.1$, (Cinco-Ley, H. and Samaniego,1977).

Figura 5.5 - Índice adimensional da produtividade em função do coeficiente adimensional de condutividade da fratura com número de propante $N_{P}>$ 0.1, (Cinco-Ley, H. and Samaniego,1977)

Figura 5.6 - Retroanálise para o modelo de cunha livre considerando propante (bauxita) de diâmetro 30/60.

Figura 5.7 - Retroanálise para o modelo semi-mecânico considerando propante (bauxita) de diâmetro 16/30. nos poços onde houve produção de propante.

Figura 5.8 - Retroanálise para o modelo semi-mecânico considerando propante (bauxita) de diâmetro 12/20 nos poços onde houve produção de propante.

Figura 5.9- Retroanálise para o modelo semi-mecânico considerando propante (bauxita) de diâmetro 16/30 nos poços onde não houve produção de propante (losangos).

Figura 5.10 - Retroanálise para o modelo do consórcio Stimlab considerando propante (bauxita) de diâmetro 16/30. Os losangos se referem a poços onde não ocorreu produção do material de sustentação da fratura.

Figura 5.11 - Retroanálise para o modelo do consórcio Stimlab considerando propante (bauxita) de diâmetro 12/20. Os losangos se referem a poços onde não ocorreu produção do material de sustentação da fratura.

Figura 5.12 - Retroanálise para o modelo de potência considerando propante (bauxita) de diâmetro 16/30. Os losangos se referem a poços onde não ocorreu produção do material de sustentação da fratura. 103 
Figura 5.13 - Retroanálise para o modelo de potência considerando propante (bauxita) de diâmetro 12/20. Os losangos se referem a poços onde não ocorreu produção do material de sustentação da fratura. 103 Figura 5.14 - Condição de estabilidade do propante (bauxita 30/60) de acordo com o modelo da cunha livre nos poços onde houve refluxo do material de sustentação.

Figura 5.15 - Condição de estabilidade do propante (bauxita 30/60) de acordo com o modelo da cunha livre nos poços onde não houve refluxo do material de sustentação.

Figura 5.16 - Condição de estabilidade do propante (bauxita 16/30 e 12/20) de acordo com o modelo semi-mecânico nos poços onde houve refluxo do material de sustentação.

Figura 5.17 - Condição de estabilidade do propante (bauxita 16/30 e 12/20) de acordo com o modelo semi-mecânico nos poços onde não houve refluxo do material de sustentação.

Figura 5.18 - Condição de estabilidade do propante (bauxita 16/30 e 12/20) de acordo com o modelo do consórcio Stimlab nos poços onde houve refluxo do material de sustentação.

Figura 5.19 - Condição de estabilidade do propante (bauxita 16/30 e 12/20) de acordo com o modelo do consórcio Stimlab nos poços onde não houve refluxo do material de sustentação. 


\section{Lista de tabelas}

Tabela 2.1 - Densidade e resistência de tipos de propante.

Tabela 2.2 - Valores de massa específica dos grãos e da massa específica aparente dos principais tipos de propante (apud www.carboceramics.com).

Tabela 2.5 - Ensaio de esmagamento em areia Otawa 20/40 e com misturas areia + DIP (15\%, 25\%) - Rae, P. et al., 2001.

Tabela 2.4 - Ensaio de esmagamento em areia Otawa 20/40 sob tensão estática de 5.000 psi (34 MPa) e 20 ciclos de carregamento entre 1.000 psi a 5.000 psi (7MPa a $34 \mathrm{MPa}$ - Rae, P. et al., 2001.

Tabela 3.1 - Equivalência entre a largura normalizada da fratura e a concentração do propante por área tratada da fratura (Milton-Tayler et al., 1992).

Tabela 4.1 - Ângulo de repouso para diferentes propantes de acordo com o consórcio Stimlab (1996-2002).

Tabela 4.2 - Valores típicos da resistência nominal do propante SMAx (Canon, 2003)

Tabela 5.1 - Poços onde a produção de propante foi observada.

Tabela 5.2 - Poços onde a produção de propante não foi observada. 91

Tabela 5.3 - Porcentagem de finos em propante de bauxita.

Tabela 5.4 - Parâmetros da fratura e resultados obtidos com o modelo de cunha livre (bauxita 30/60).

Tabela 5.5 - Parâmetros da fratura e resultados obtidos com o modelo semi-mecânico (bauxita 16/30)

Tabela 5.6 - Parâmetros da fratura e resultados obtidos com o modelo semi-mecânico (bauxita 12/20)

Tabela 5.7 - Parâmetros da fratura e resultados obtidos com o modelo do consórcio Stimlab (bauxita 16/30)

Tabela 5.8 - Parâmetros da fratura e resultados obtidos com o modelo do consórcio Stimlab (bauxita 12/20). 
Tabela 5.9 - Parâmetros da fratura e resultados obtidos com o modelo de potência nos poços onde ocorreu produção de propante. 114 Tabela 5.10 - Parâmetros da fratura e resultados obtidos com o modelo de potência nos poços onde não ocorreu produção de propante. 


\section{LISTA DE SÍMBOLOS}

Parâmetros da fratura e resultados obtidos com o modelo de potência nos poços onde não ocorreu produção de propante.

\section{Alfabeto Latino}

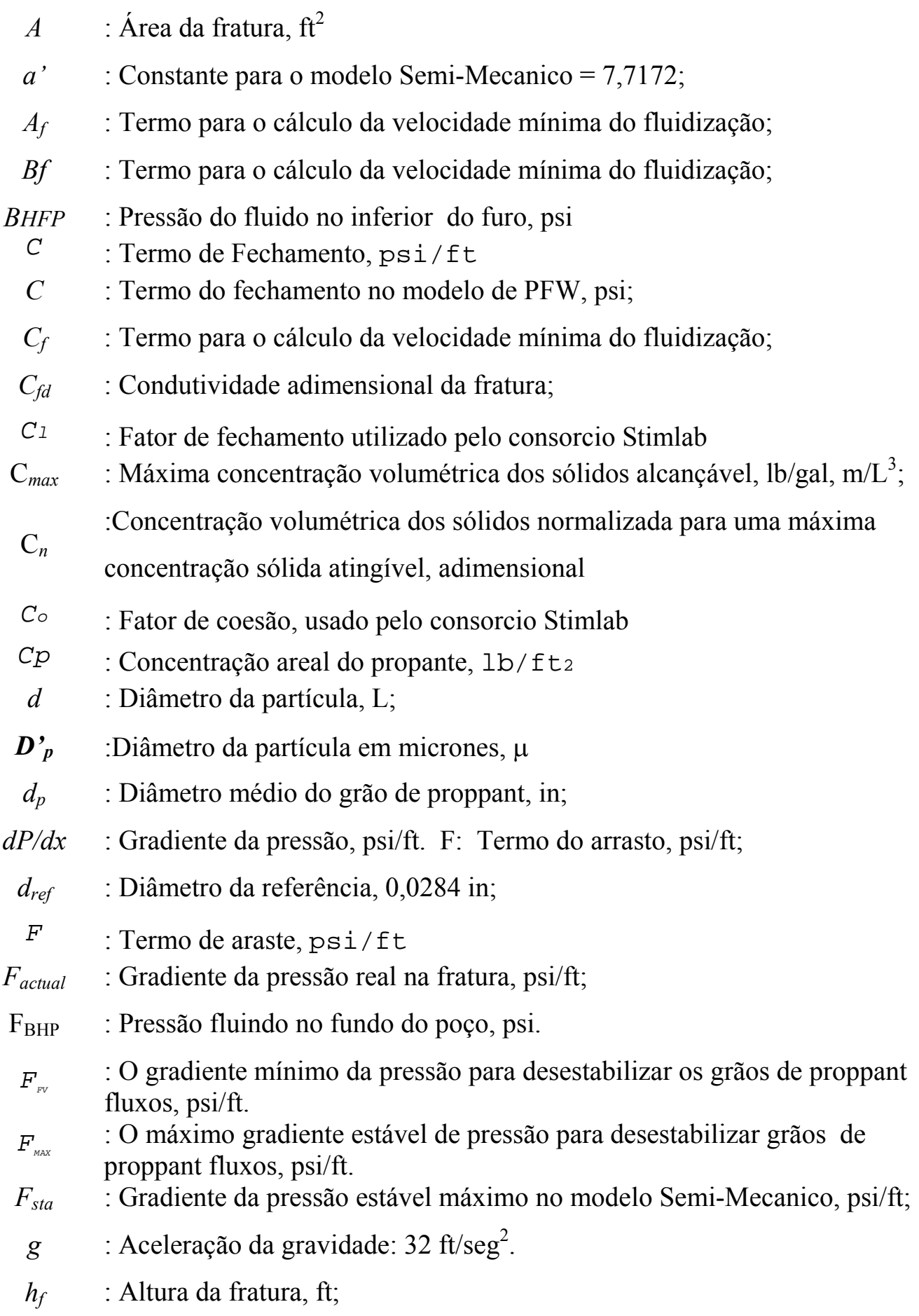




$$
\begin{aligned}
& J_{d, i d} \quad \text { : Ótimo índice adimensional de produtividade } \\
& h_{p} \quad \text { : Espessura neta de produção, } \mathrm{ft} \text {; } \\
& K \quad \text { : È a permeabilidade em (Darcy) } \\
& k \quad \text { : Permeabilidade da fartura, md } \\
& \text { kf : permeabilidade do pacote de propante, md } \\
& k_{n} \quad \text { : Constante da mola Normal; } \\
& L \quad \text { : È a longitude da fratura em, (cm); } \\
& m \quad \text { : Massa do grão; } \\
& \mathrm{M}_{\mathrm{P}} \quad \text { : Massa de proppant, lb; } \\
& M_{P F B} \quad \text { : Massa de proppant que fluído para trás, libras; } \\
& \tilde{n} \quad \text { : Vetor da unidade no sentido normal; } \\
& P \quad \text { : É a queda da pressão na fratura em (atm) } \\
& P_{b h} \quad \text { : Pressão de fluxo no fundo do poço, psi ; } \\
& P_{c, b h} \text { : Pressão de fechamento no fundo do poço, psi ; } \\
& P_{c, \text { net }} \quad \text { : Pressão efetiva do fechamento, psi; } \\
& P F \quad: \text { Factor proppant } \\
& \text { : Pressão final da formação (para estes cálculos supostos para ser zero } \\
& \text { para resultados conservadores); } \\
& \text { : Pressão inicial da formação (na pressão do fechamento do tempo foi } \\
& \text { medido); } \\
& \Delta P_{p} \quad \text { : Pressão efetiva } \\
& P_{r d} \quad \text { : Pressão de depleção do reservatório. } \\
& P_{r i} \quad \text { : Pressão inicial do reservatório (psi), } \\
& P_{w f} \quad \text { : Pressão no fundo do poço } \\
& q_{\text {crit }} \quad \text { : É o fluxo crítico, } \mathrm{ft}^{3} / \mathrm{seg} \\
& Q_{f} \quad \text { : Taxa volumétrico do fluxo do fluido } \mathrm{em}^{3} / \mathrm{s} \\
& \text { Qtotal : Taxa de fluxo total, scf/D } \\
& \text { Re : Numero de Reynolds } \\
& \text { Rf : Numero de Reynolds do fluido } \\
& R_{f} t \quad \text { : número Reynolds crítico no modelo de Carreau } \\
& R_{G} \quad \text { : Número de Reynolds da gravidade } \\
& S G_{p} \quad \text { : Gravidade específica de proppant; } \\
& S_{M A X} \quad \text { : Força máxima de proppant, psi; } \\
& S_{t} \quad \text { : Termo da resistência; } \\
& V \quad: \text { È a velocidade do fluido em }(\mathrm{cm} / \mathrm{sec}) \\
& V_{c} \quad: \text { Velocidade critica do consorcio Stimlab, } \mathrm{ft} / \mathrm{seg} ;
\end{aligned}
$$




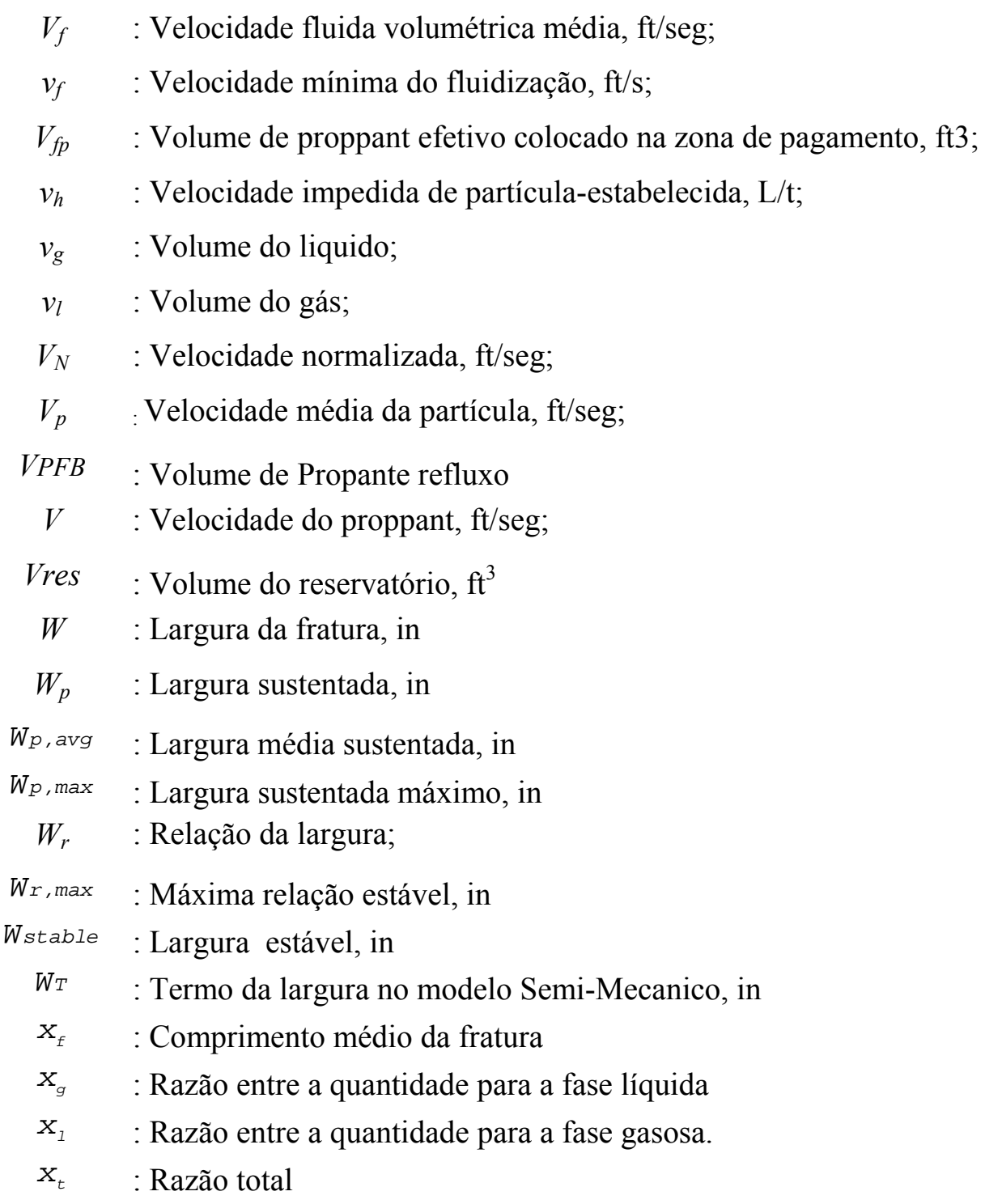

\section{Alfabeto grego}

$\beta \quad$ : Fator ou o coeficiente da resistência inercial.

$\varepsilon_{m f} \quad$ : Densidade média de vácuos da fase de fluidização mínimo;

$\mu_{f} \quad$ : Viscosidade do fluido, cp;

$\mu_{a} \quad$ : Viscosidade aparente da pasta, cp;

$\mu_{o} \quad$ : Viscosidade normalizada do fluido limpo, cp;

$\mu_{g} \quad$ : Viscosidade do fluido;

$\mu_{l} \quad$ : Viscosidade do gás;

$\rho_{f} \quad$ : Densidade do fluido, $\mathrm{lb} / \mathrm{ft}^{3}$;

$\rho_{p} \quad$ : Densidade da partícula, $\mathrm{lb} / \mathrm{ft}^{3}$; 


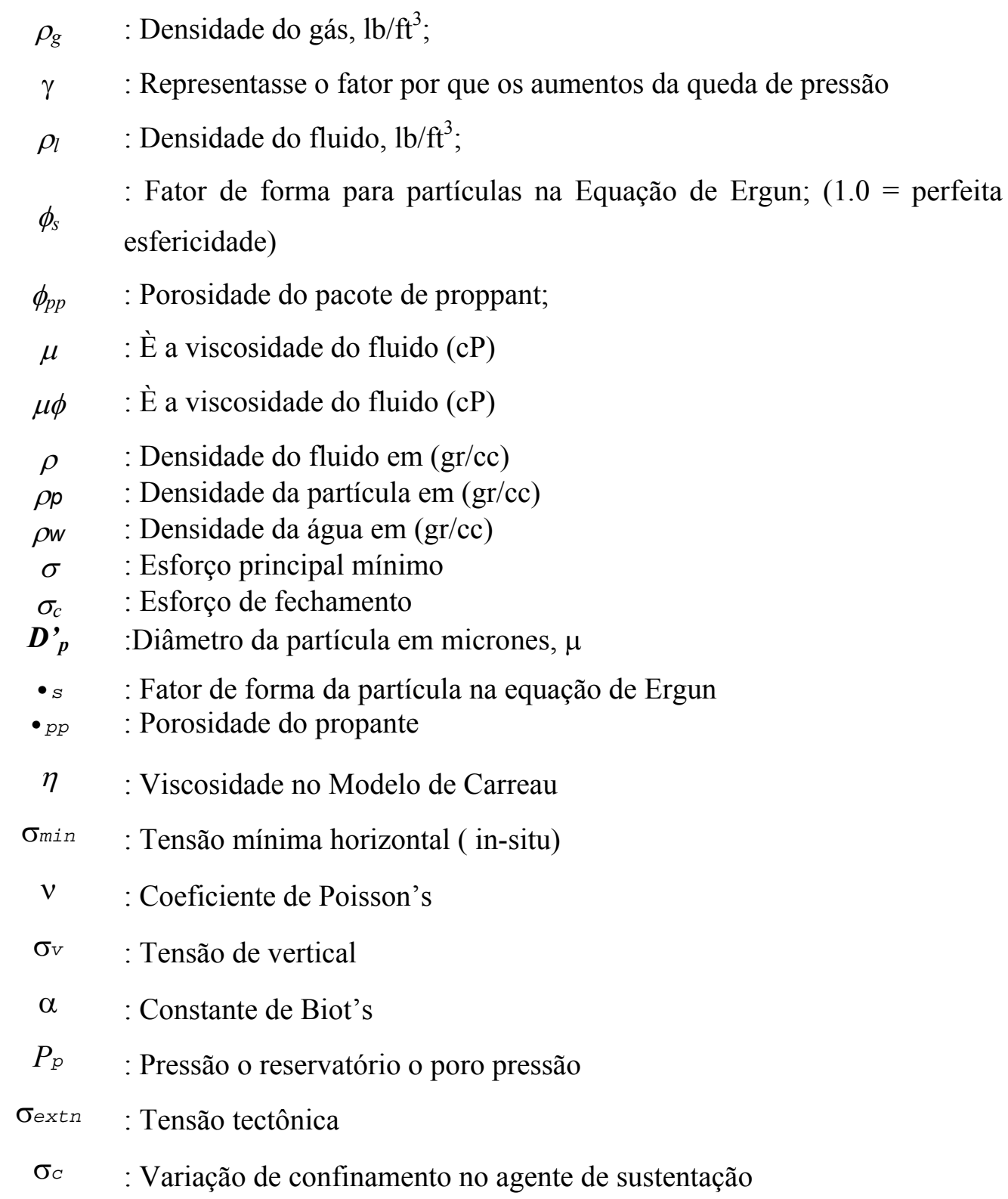

